\title{
Meanings Of Weighted Beta Capm Index And Beta Derminants Of Vietcombank In Vietnam During Pre-Low Inflation Time 2011-2016 In The Concept Of Sustainability
}

\author{
Leng Thi Lan, $\mathrm{PhD}$ \\ Thai Nguyen University of Agriculture and Forestry, Vietnam \\ lengthilan@tuaf.edu.vn \\ Dinh Tran Ngoc Huy, MBA, PhD candidate (corresponding) \\ Banking University HCMC, Ho Chi Minh city Vietnam \\ dtnhuy2010@gmail.com \\ Nguyen Dinh Trung, $\mathrm{PhD}$ \\ National Economics University (NEU), Vietnam \\ trungnd@neu.edu.vn \\ Nguyen Thi Hang, $\mathrm{PhD}$ \\ Thai Nguyen University, University of Information and Communication Technology, \\ Vietnam \\ nthang@ictu.edu.vn
}

\begin{abstract}
The aim of this study is to figure out the meanings of weighted beta index in bank sector and beta determinants of Vietcombank -VCB during pre-low (L) inflation period 2011-2016.

In reality Vietcombank has gained many achievements but also there are certain weaknesses. Hung and Liu (2005) tested the volatility of airline betas in the capital asset pricing and three-factor pricing models, as well as exploring the potential factors affecting their values. While Fama and Frech (2004) suggest to add firm size into traditional formula of beta CAPM.

The study results tell us that Market risk can increased and This may be caused by the increase in lending rate and decrease in both CPI and G (from our regression OLS).

Therefore, governmental agencies need to reduce lending rate and increase GDP growth and not decreasing much CPI.
\end{abstract}

Key words: pre-low inflation, bank sustainability, Vietnam banks, beta CAPM, economic development

JEL: M21, G30, G32, G38

\section{Introduction}

First, we recognize the importance of digital technology in banking also increase to a new level in recent years. 
Once again, Fama and French (2004) confirmed that beta CAPM, developed by Sharpe (1964) and Lintner (1965) had wide applications in evaluating performance of managed portfolios.

Next, We emphasize that the role of new concept of bank sustainable development or banking sustainability in relation to risk management approach.

And the below chart 1 show us that beta VCB is little higher than weighted beta index.

Chart 1 - Movement of beta VCB and weighted beta

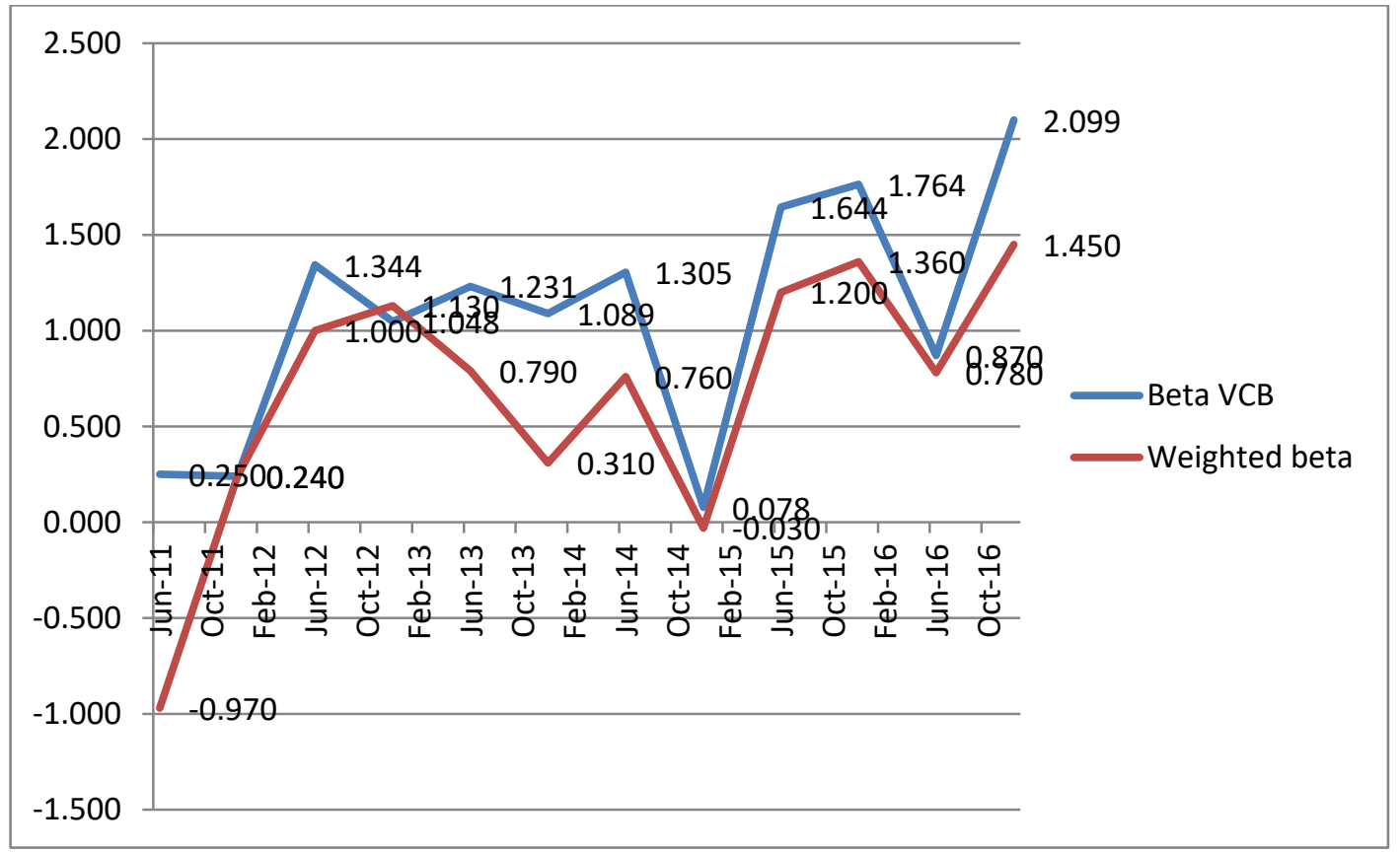

(source: authors calculation and stock exchange)

\section{Research question:}

What are beta determinants and comparison effects on weighted beta index with beta

CAPM of Vietcombank - VCB?

\section{Literature review}

First, Melicher (1974) said that Research in portfolio management area and capital markets led to development of a market risk fundamental concept.

Then, We summarize previous studies as follows:

Table 1 - Summary of previous studies

\begin{tabular}{|l|l|l|}
\hline Authors & Year & Contents, results \\
\hline Hung and Liu & 2005 & $\begin{array}{l}\text { The beta value, an indicator of } \\
\text { systematic risk, is used to estimate } \\
\text { the costs of equity and the evaluation } \\
\text { of a stock's reasonable price. It is } \\
\text { useful to airlines because their capital } \\
\text { assets and operations are relatively }\end{array}$ \\
\hline
\end{tabular}




\begin{tabular}{|c|c|c|}
\hline & & $\begin{array}{l}\text { sensitive to systematic risks. To } \\
\text { obtain better estimates, it is useful to } \\
\text { identify the causes for fluctuations in } \\
\text { beta values. This The findings } \\
\text { suggest that airline betas are volatile } \\
\text { over time and that crashes and stock } \\
\text { market trends may also impact them. } \\
\text { The business cycle, operating and } \\
\text { financial leverage, and capital } \\
\text { structure all positively influence the } \\
\text { sample airlines' betas as well. The } \\
\text { effects of return on equity on betas } \\
\text { are negative, and liquidity might also } \\
\text { affect betas. }\end{array}$ \\
\hline Karim, A.J & 2011 & $\begin{array}{l}\text { Management Information Systems } \\
\text { (MIS) is the key factor to facilitate } \\
\text { and attain efficient decision making } \\
\text { in an organization. }\end{array}$ \\
\hline Blanco & 2012 & $\begin{array}{l}\text { tests the American NYSE market, } \\
\text { the expected returns of a portfolios } \\
\text { selection according to the CAPM and } \\
\text { Fama and French Three Factor } \\
\text { Model. The portfolios have been } \\
\text { constructed according to the size and } \\
\text { BV/MV.Empirical results point out } \\
\text { that Fama and French Three Factor } \\
\text { Model is better than CAPM } \\
\text { according to the goal of explaining } \\
\text { the expected returns of the portfolios }\end{array}$ \\
\hline Satrio & 2015 & $\begin{array}{l}\text { Relationship between expected } \\
\text { return, the size of the firm, and the } \\
\text { firm's value empirically tested in this } \\
\text { study, with testing in developing } \\
\text { countries, namely the Indonesian } \\
\text { capital market. This study seeks to } \\
\text { test the CAPM model that proposed } \\
\text { by Sharpe (1964), Lintner (1965), } \\
\text { Mossin (1966), and three-factor } \\
\text { model of Fama and French (1993). } \\
\text { The results showed that CAPM is } \\
\text { alive and well, and the three-factor } \\
\text { model is a powerful model for } \\
\text { explaining the stock returns in } \\
\text { Indonesia and provide a better } \\
\text { explanation. }\end{array}$ \\
\hline Huy, D.T.N, Loan, & 2020 & Suggest build risk model under \\
\hline
\end{tabular}




\begin{tabular}{|l|l|l|}
\hline B.T.T, \& Anh, P.T & 2019 & $\begin{array}{l}\text { macro effects for Vietcombank stock } \\
\text { price in Vietnam }\end{array}$ \\
\hline Gupta & $\begin{array}{l}\text { In various areas of bank functions } \\
\text { including finance, HR, etc. IS - } \\
\text { information system roles are } \\
\text { important to serve for client relation. }\end{array}$ \\
\hline $\begin{array}{l}\text { Grabowska and } \\
\text { Orlowski }\end{array}$ & 2020 & $\begin{array}{l}\text { market risk should play a stronger } \\
\text { role in macroeconomic modeling and } \\
\text { forecasting than it has been } \\
\text { recognized thus far in the literature }\end{array}$ \\
\hline
\end{tabular}

\section{Methodology}

\section{Method and Data}

This study mainly use combination of quantitative methods and qualitative methods including synthesis, inductive and explanatory methods.

For quantitative analysis, the study is supported with OLS regression.

All internet data such as stock price, exchange rate, inflation, GDP growth, risk free rate we take from reliable internet data sources, esp. from website of State Bank of Vietnam, Bureau of Statistics, Ministry of Finance, banks, etc.

\section{Looking at statistics below, we see:}

- We experience standard dev get highest values in case of exchange rate and SP500 (figure 1)

- We experience Correlation b.t trade balance and weighted beta higher than that between beta and SP500 (figure 2)

- Highest values of standard deviation belong to: VNIndex (figure 3)

- Correlation between IM and beta VCB lower than that between beta and R (figure 4)

\section{Figure 1- VCB and external descriptive}

\begin{tabular}{|l|c|c|c|c|}
\hline & BETA_VCB & EX RATE & SP500 & TRADEBA $\ldots$ \\
\hline Mean & 0.951417 & 21966.92 & 1785.692 & -243.5000 \\
\hline Median & 1.068500 & 22030.00 & 1856.630 & -162.5000 \\
\hline Maximum & 1.764000 & 23230.00 & 2238.830 & 498.0000 \\
\hline Minimum & 0.078000 & 20618.00 & 1292.280 & -1162.000 \\
\hline Std. Dev. & 0.560118 & 831.8935 & 355.6315 & 430.5632 \\
\hline Skewness & -0.235542 & -0.184123 & -0.245780 & -0.353671 \\
\hline Kurtosis & 1.830915 & 1.779555 & 1.481990 & 3.242509 \\
\hline & & & & \\
\hline Jarque-Bera & 0.794341 & 0.812546 & 1.272993 & 0.279572 \\
\hline Probability & 0.672220 & 0.666128 & 0.529143 & 0.869545 \\
\hline & & & & \\
\hline Sum & 11.41700 & 263603.0 & 21428.30 & -2922.000 \\
\hline Sum Sq. Dev. & 3.451055 & 7612515. & 1391211. & 2039231. \\
\hline
\end{tabular}

(source: author calculation and stock exchange)

Figure 2- VCB and external correlation 


\begin{tabular}{|c|c|c|c|c|}
\hline \multicolumn{5}{|c}{ Correlation Matrix } \\
\hline & BETA_VCB & EX_RATE & \multicolumn{1}{c|}{ SP500 } & TRADEBA... \\
\hline BETA_VCB & 1.000000 & -0.011462 & 0.224229 & 0.338854 \\
\hline EX_RATE & -0.011462 & 1.000000 & 0.540064 & -0.495971 \\
\hline SP500 & 0.224229 & 0.540064 & 1.000000 & -0.437273 \\
\hline TRADEBA $\ldots$ & 0.338854 & -0.495971 & -0.437273 & 1.000000 \\
\hline
\end{tabular}

(source: author calculation and stock exchange)

Figure 3- VCB and internal descriptive

\begin{tabular}{|l|c|c|c|c|c|c|c|}
\hline & BETA_VCB & CPI & G & IM & R & RF & VNIINDEX \\
\hline Mean & 0.951417 & 0.062275 & 0.057550 & 157.9250 & 0.127917 & 0.071463 & 516.5733 \\
\hline Median & 1.068500 & 0.053900 & 0.056500 & 163.9000 & 0.115000 & 0.063500 & 525.1300 \\
\hline Maximum & 1.764000 & 0.181300 & 0.068100 & 194.8000 & 0.190000 & 0.132000 & 664.8700 \\
\hline Minimum & 0.078000 & 0.006300 & 0.043800 & 117.4000 & 0.100000 & 0.046000 & 351.5500 \\
\hline Std. Dev. & 0.560118 & 0.056352 & 0.007277 & 24.95659 & 0.030411 & 0.022599 & 97.65861 \\
\hline Skewness & -0.235542 & 1.157131 & -0.270524 & -0.135918 & 1.073834 & 1.756510 & -0.132524 \\
\hline Kurtosis & 1.830915 & 3.217708 & 2.260704 & 1.845200 & 2.812611 & 5.433403 & 1.865595 \\
\hline & & & & & & & \\
\hline Jarque-Bera & 0.794341 & 2.701604 & 0.419646 & 0.703729 & 2.323797 & 9.131377 & 0.678563 \\
\hline Probability & 0.672220 & 0.259032 & 0.810728 & 0.703375 & 0.312892 & 0.010403 & 0.712282 \\
\hline & & & & & & & \\
\hline Sum & 11.41700 & 0.747300 & 0.690600 & 1895.100 & 1.535000 & 0.857550 & 6198.880 \\
\hline Sum Sq. Dev. & 3.451055 & 0.034931 & 0.000582 & 6851.142 & 0.010173 & 0.005618 & 104909.3 \\
\hline
\end{tabular}

(source: author calculation and stock exchange)

Figure 4- VCB and internal correlation

\begin{tabular}{|c|c|c|r|r|r|r|r|}
\hline \multicolumn{9}{|c|}{ Correlation Matrix } \\
\hline & BETA_VCB & CPI & \multicolumn{1}{c|}{ G } & \multicolumn{1}{c|}{ IM } & \multicolumn{1}{c|}{ R } & \multicolumn{1}{c|}{ RF } & VNIINDEX \\
\hline BETA_VCB & 1.000000 & -0.630923 & -0.362034 & -0.340323 & -0.027243 & -0.313611 & 0.248715 \\
\hline CPI & -0.630923 & 1.000000 & 0.055118 & 0.386897 & 0.464299 & 0.597007 & -0.788603 \\
\hline G & -0.362034 & 0.055118 & 1.000000 & 0.508115 & 0.065833 & -0.425075 & 0.181795 \\
\hline IM & -0.340323 & 0.386897 & 0.508115 & 1.000000 & 0.454293 & 0.037760 & -0.264605 \\
\hline R & -0.027243 & 0.464299 & 0.065833 & 0.454293 & 1.000000 & 0.033608 & -0.765116 \\
\hline RF & -0.313611 & 0.597007 & -0.425075 & 0.037760 & 0.033608 & 1.000000 & -0.469239 \\
\hline VNIINDEX & 0.248715 & -0.788603 & 0.181795 & -0.264605 & -0.765116 & -0.469239 & 1.000000 \\
\hline
\end{tabular}

(source: author calculation and stock exchange)

Figure 5- weighted beta and external descriptive

\begin{tabular}{|l|r|r|r|r|}
\hline & WEIGHTE... & EX RATE & \multicolumn{1}{c|}{ SP500 } & TRADEBA $\ldots$. \\
\hline Mean & 0.668333 & 21966.92 & 1785.692 & -243.5000 \\
\hline Median & 0.785000 & 22030.00 & 1856.630 & -162.5000 \\
\hline Maximum & 1.450000 & 23230.00 & 2238.830 & 498.0000 \\
\hline Minimum & -0.970000 & 20618.00 & 1292.280 & -1162.000 \\
\hline Std. Dev. & 0.688514 & 831.8935 & 355.6315 & 430.5632 \\
\hline Skewness & -1.095298 & -0.184123 & -0.245780 & -0.353671 \\
\hline Kurtosis & 3.633148 & 1.779555 & 1.481990 & 3.242509 \\
\hline & & & & \\
\hline Jarque-Bera & 2.599794 & 0.812546 & 1.272993 & 0.279572 \\
\hline Probability & 0.272560 & 0.666128 & 0.529143 & 0.869545 \\
\hline & & & & \\
\hline Sum & 8.020000 & 263603.0 & 21428.30 & -2922.000 \\
\hline Sum Sq. Dev. & 5.214567 & 7612515. & 1391211. & 2039231. \\
\hline
\end{tabular}


(source: author calculation and stock exchange)

Figure 6- weighted beta and external correlation

\begin{tabular}{c|c|c|c|c|}
\hline \hline \multicolumn{5}{c}{ Correlation Matrix } \\
\hline & WEIGHTE $\ldots$ & EX_RATE & SP500 & TRADEBA ... \\
\hline WEIGHTE ... & 1.000000 & 0.322915 & 0.470435 & 0.211795 \\
\hline EX_RATE & 0.322915 & 1.000000 & 0.540064 & -0.495971 \\
\hline SP500 & 0.470435 & 0.540064 & 1.000000 & -0.437273 \\
\hline TRADEBA ... & 0.211795 & -0.495971 & -0.437273 & 1.000000 \\
\hline
\end{tabular}

(source: author calculation and stock exchange)

Figure 7- weighted beta and internal descriptive

\begin{tabular}{|l|c|c|c|c|c|c|c|}
\hline & WEIGHTE $\ldots$ & CPI & G & IM & R & RF & VNIINDEX \\
\hline Mean & 0.668333 & 0.062275 & 0.057550 & 157.9250 & 0.127917 & 0.071463 & 516.5733 \\
\hline Median & 0.785000 & 0.053900 & 0.056500 & 163.9000 & 0.115000 & 0.063500 & 525.1300 \\
\hline Maximum & 1.450000 & 0.181300 & 0.068100 & 194.8000 & 0.190000 & 0.132000 & 664.8700 \\
\hline Minimum & -0.970000 & 0.006300 & 0.043800 & 117.4000 & 0.100000 & 0.046000 & 351.5500 \\
\hline Std. Dev. & 0.688514 & 0.056352 & 0.007277 & 24.95659 & 0.030411 & 0.022599 & 97.65861 \\
\hline Skewness & -1.095298 & 1.157131 & -0.270524 & -0.135918 & 1.073834 & 1.756510 & -0.132524 \\
\hline Kurtosis & 3.633148 & 3.217708 & 2.260704 & 1.845200 & 2.812611 & 5.433403 & 1.865595 \\
\hline & & & & & & & \\
\hline Jarque-Bera & 2.599794 & 2.701604 & 0.419646 & 0.703729 & 2.323797 & 9.131377 & 0.678563 \\
\hline Probability & 0.272560 & 0.259032 & 0.810728 & 0.703375 & 0.312892 & 0.010403 & 0.712282 \\
\hline & & & & & & & \\
\hline Sum & 8.020000 & 0.747300 & 0.690600 & 1895.100 & 1.535000 & 0.857550 & 6198.880 \\
\hline Sum Sq. Dev. & 5.214567 & 0.034931 & 0.000582 & 6851.142 & 0.010173 & 0.005618 & 104909.3 \\
\hline
\end{tabular}

(source: author calculation and stock exchange)

Figure 8- weighted beta and internal correlation

\begin{tabular}{|c|r|r|r|r|r|r|r|}
\hline \multicolumn{9}{|c|}{ Correlation Matrix } \\
\hline & WEIGHTE $\ldots$ & CPI & G & IM & R & \multicolumn{1}{c|}{ RF } & VNIINDEX \\
\hline WEIGHTE $\ldots$ & 1.000000 & -0.656105 & 0.130500 & 0.110509 & 0.051269 & -0.660912 & 0.443081 \\
\hline CPI & -0.656105 & 1.000000 & 0.055118 & 0.386897 & 0.464299 & 0.597007 & -0.788603 \\
\hline G & 0.130500 & 0.055118 & 1.000000 & 0.508115 & 0.065833 & -0.425075 & 0.181795 \\
\hline IM & 0.110509 & 0.386897 & 0.508115 & 1.000000 & 0.454293 & 0.037760 & -0.264605 \\
\hline R & 0.051269 & 0.464299 & 0.065833 & 0.454293 & 1.000000 & 0.033608 & -0.765116 \\
\hline RF & -0.660912 & 0.597007 & -0.425075 & 0.037760 & 0.033608 & 1.000000 & -0.469239 \\
\hline VNIINDEX & 0.443081 & -0.788603 & 0.181795 & -0.264605 & -0.765116 & -0.469239 & 1.000000 \\
\hline
\end{tabular}

(source: author calculation and stock exchange)

\section{Main results}

\subsection{Overall results}

As seeing in below charts, we find that:

- Between CPI and beta: there is negative relation (chart 2)

- Between lending rate and beta : there are positive relation in case weighted beta, but negative in case beta $\mathrm{VCB}$ (chart 1)

- Between VNIndex and beta: there is also positive relation (chart 3)

\section{Chart 2 - R and two betas estimated}




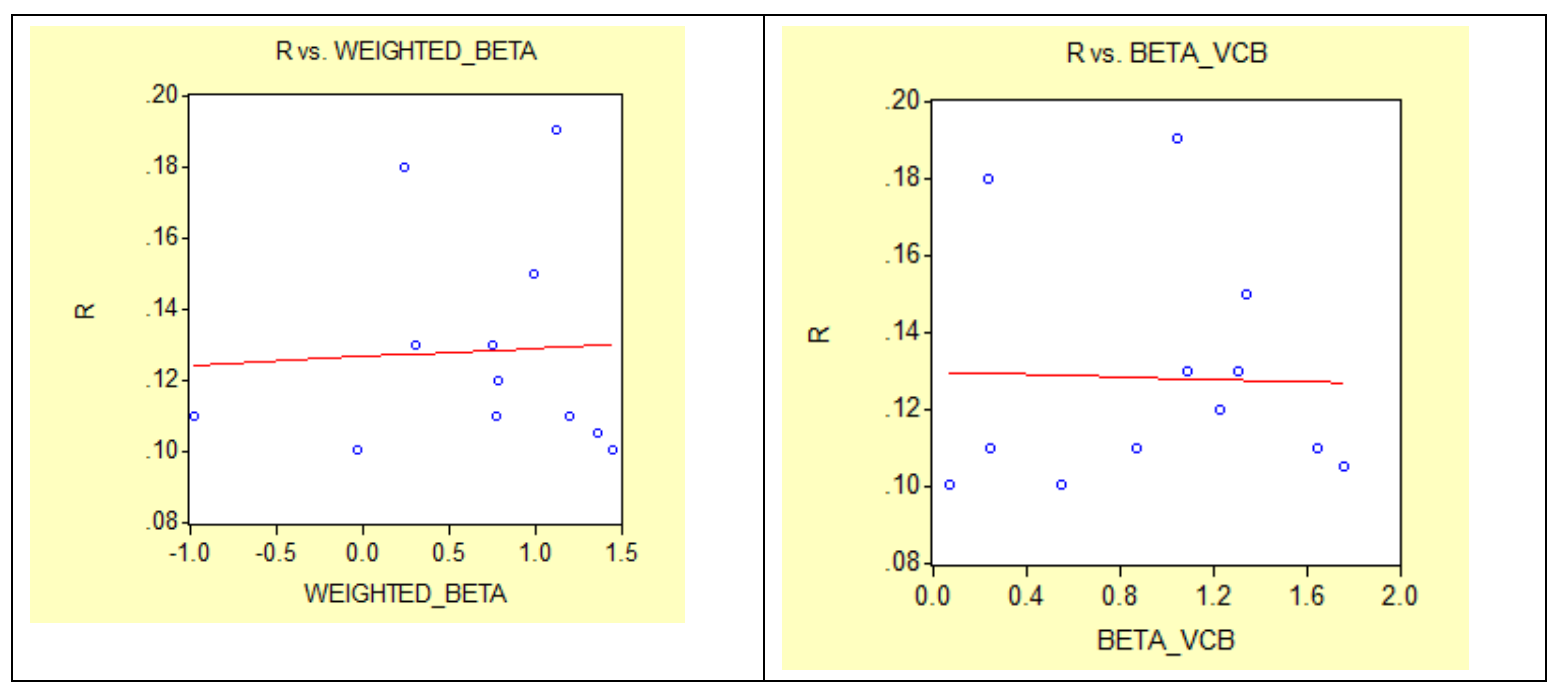

(source: author calculation and stock exchange)

Chart 3 - CPI and two betas estimated

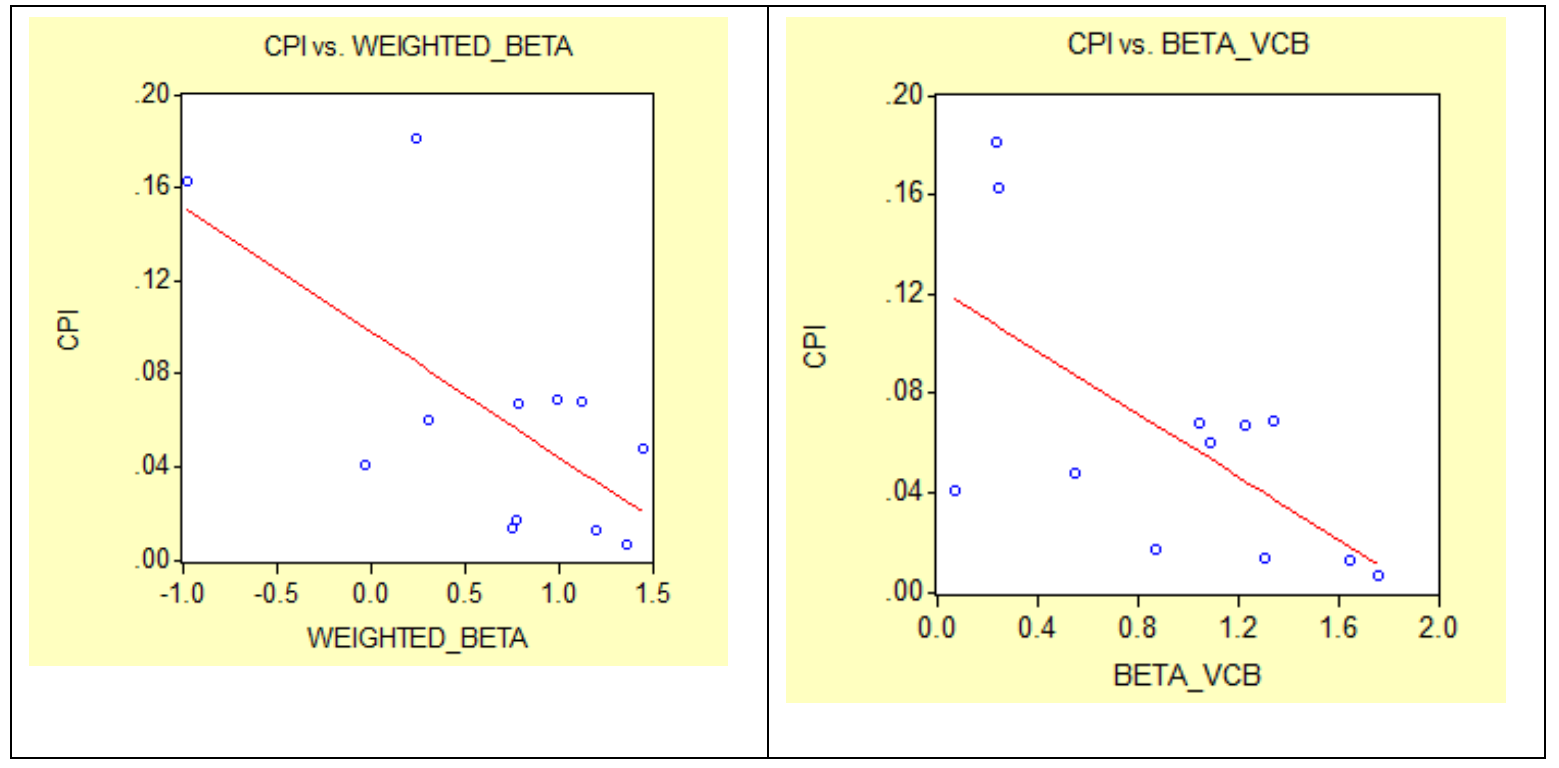

(source: author calculation and stock exchange)

\section{Chart 4 - VNIndex and two betas estimated}




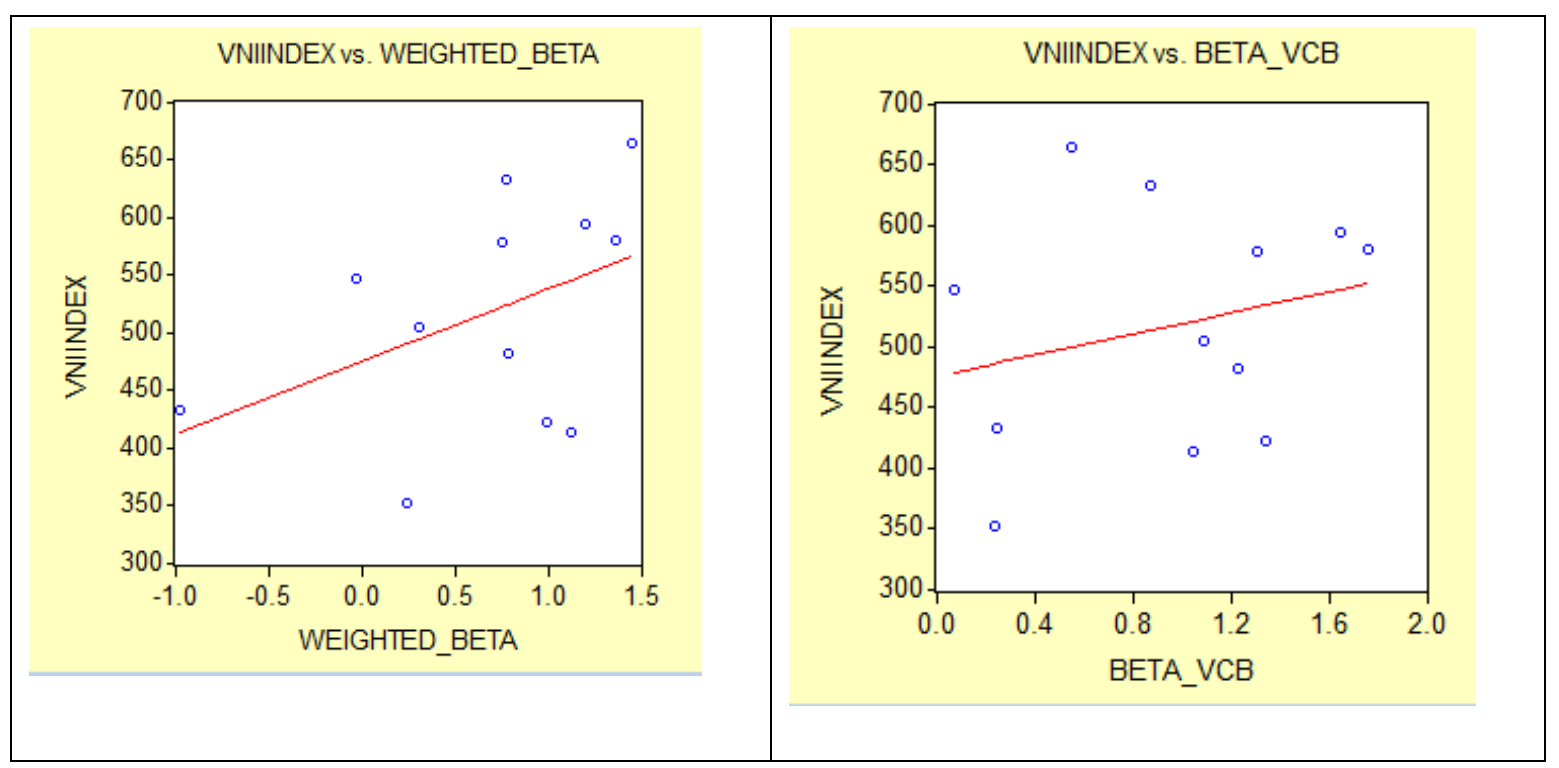

(source: author calculation and stock exchange)

\subsection{OLS Regression results}

In below section, we have result of OLS for 1 factor and see that:

- because coefficient calculated of -8.01, CPI and weighted beta 2011-16 has negative correlation (see figure 9)

- because coefficient calculated of 1.16, $\mathrm{R}$ and weighted beta 2011-16 has positive correlation (see figure 10)

- because coefficient calculated of 0.003, IM and weighted beta 2011-16 has positive correlation (see figure 11)

\section{Figure 9 - OLS for CPI}

\section{Weighted beta 11-16}

Dependent Variable: WEIGHTED_BETA

Method: Least Squares

Date: 07/28/21 Time: 11:26

Sample: 112

Included observations: 12

\begin{tabular}{lrlll}
\hline \hline \multicolumn{1}{c}{ Variable } & Coefficient & Std. Error & t-Statistic & Prob. \\
\hline \hline \multicolumn{1}{c}{ CPI } & -8.016351 & 2.915816 & -2.749265 & 0.0205 \\
C & 1.167552 & 0.240252 & 4.859705 & 0.0007 \\
\hline \hline R-squared & 0.430474 & Mean dependent var & 0.668333 \\
Adjusted R-squared & 0.373521 & S.D. dependent var & 0.688514 \\
S.E. of regression & 0.544962 & Akaike info criterion & 1.774809 \\
Sum squared resid & 2.969832 & Schwarz criterion & 1.855627 \\
Log likelihood & -8.648855 & F-statistic & 7.558456 \\
Durbin-Watson stat & 1.591600 & Prob(F-statistic) & 0.020504 \\
\hline \hline
\end{tabular}

(source: author calculation and stock exchange) 
Figure 10 - OLS for $\mathbf{R}$

Dependent Variable: WEIGHTED_BETA

Method: Least Squares

Date: 07/28/21 Time: 11:26

Sample: 112

Included observations: 12

\begin{tabular}{lrlll}
\hline \hline \multicolumn{1}{c}{ Variable } & Coefficient & Std. Error & t-Statistic & Prob. \\
\hline \hline R & 1.160762 & 7.150145 & 0.162341 & 0.8743 \\
C & 0.519853 & 0.938017 & 0.554204 & 0.5916 \\
\hline \hline R-squared & 0.002629 & Mean dependent var & 0.668333 \\
Adjusted R-squared & -0.097109 & S.D. dependent var & 0.688514 \\
S.E. of regression & 0.721170 & Akaike info criterion & 2.335128 \\
Sum squared resid & 5.200860 & Schwarz criterion & 2.415946 \\
Log likelihood & -12.01077 & F-statistic & 0.026355 \\
Durbin-Watson stat & 1.021743 & Prob(F-statistic) & 0.874271 \\
\hline \hline
\end{tabular}

(source: author calculation and stock exchange)

Figure 11 - OLS for IM

Dependent Variable: WEIGHTED_BETA

Method: Least Squares

Date: 07/28/21 Time: 11:27

Sample: 112

Included observations: 12

\begin{tabular}{lrlll}
\hline \hline \multicolumn{1}{c}{ Variable } & Coefficient & Std. Error & t-Statistic & Prob. \\
\hline \hline \multicolumn{1}{c}{ IM } & 0.003049 & 0.008671 & 0.351612 & 0.7324 \\
C & 0.186858 & 1.384922 & 0.134923 & 0.8953 \\
\hline \hline R-squared & 0.012212 & Mean dependent var & 0.668333 \\
Adjusted R-squared & -0.086567 & S.D. dependent var & 0.688514 \\
S.E. of regression & 0.717697 & Akaike info criterion & 2.325472 \\
Sum squared resid & 5.150886 & Schwarz criterion & 2.406290 \\
Log likelihood & -11.95283 & F-statistic & 0.123631 \\
Durbin-Watson stat & 1.064046 & Prob(F-statistic) & 0.732425 \\
\hline \hline
\end{tabular}

(source: author calculation and stock exchange)

Next, we run OLS as follows:

Table 2 - OLS for external variables pre-L inflation 2011-2016 period

\begin{tabular}{|l|l|l|l|}
\hline & \multicolumn{3}{|c|}{ Coefficients } \\
\hline & $\begin{array}{l}\text { Weighted beta } \\
\text { index 2011-20 }\end{array}$ & $\begin{array}{l}\text { Weighted beta } \\
\text { index 2011-16 }\end{array}$ & Beta VCB \\
\hline Exchange rate & 0.00022 & 0.0002 & 8.39E \\
\hline SP500 & $5.11 E$ & 0.001 & 0.00072 \\
\hline Trade balance & 0.00026 & 0.0009 & 0.00070 \\
\hline
\end{tabular}




\begin{tabular}{|l|l|l|l|}
\hline SER & 0.59 & 0.56 & 0.55 \\
\hline $\begin{array}{l}\text { Akaike info } \\
\text { criteria }\end{array}$ & 1.98 & 1.97 & 1.92 \\
\hline
\end{tabular}

(source: authors calculation and stock exchange)

Table 3 - OLS for internal variables 2011-16 period

\begin{tabular}{|l|l|l|l|}
\hline & \multicolumn{3}{|c|}{ Coefficients } \\
\hline & $\begin{array}{l}\text { Weighted beta } \\
\text { index 2011-20 }\end{array}$ & $\begin{array}{l}\text { Weighted beta } \\
\text { index 2011-16 }\end{array}$ & Beta VCB \\
\hline CPI & -9.07 & -5.7 & -8.9 \\
\hline G & 7.8 & -24.2 & -21.7 \\
\hline IM & 0.004 & 0.009 & -0.0003 \\
\hline R & 7.3 & 10.02 & 4.1 \\
\hline Rf & -4.2 & 10.1 & -0.5 \\
\hline VNIndex & 0.0003 & 0.002 & -0.001 \\
\hline C & -0.78 & -1.02 & 3.07 \\
\hline R-squared & 0.62 & 0.72 & 0.61 \\
\hline SER & 0.44 & 0.53 & 0.51 \\
\hline $\begin{array}{l}\text { Akaike info } \\
\text { criteria }\end{array}$ & 1.5 & 1.86 & 1.8 \\
\hline
\end{tabular}

(source: authors calculation and stock exchange)

\section{Discussion}

\section{During pre $-\mathbf{L}$ inflation:}

In case of internal elements we find out: GDP growth and CPI have egative correlation with weighted beta and beta VCB 2011-2016, while R - lending rate has positive correlation with both beta.

Moreover, the coefficients for CPI, G and R are higher in 2011-2016.

In case of external variables we figure out: trade balance and SP500 and exchange rate, all factors have positive correlation with weighted beta index as well as beta VCB. Thats happen during post $-\mathrm{L}$ inflation stage.

Additionally, the coefficients for SP500 and beta VCB and weighted beta is higher in 2011-2016.

\section{Conclusion}

Rostov (1960) mentioned quality of economic growth include series of continuing stages in which each country has passed. And researches has mentioned limitations for process of economic growth as well as inequalities in using resources. 
In the scope of this study, we will keep bank sustainability for economic growth, if we can have better risk management with below suggested policies:

Market risk can increased and This may be caused by the increase in lending rate and decrease in both CPI and G (from our table 2 and 3 ).

Therefore, governmental agencies need to reduce lending rate and increase GDP growth and not decreasing much CPI.

Finally, Huy, D.T.N (2015) stated that we need to apply corporate governance standards such as Limited South Asian corporate governance model into corporations to reduce and control risk better.

\section{Limitation of research}

We can expand our research model for other industries and other markets. 


\section{References}

1. Aracil, E., Sanchez, J.J.N., \& Forcadel, F.J. (2021). Sustainable banking: A literature review and integrative framework, Finance Research Letters, 1. DOI:10.1016/j.frl.2021.101932

2. Blanco, B. (2012). The use of CAPM and Fama and French Three Factor Model: portfolios selection, Public and Municipal Finance, 1(2).

3. Duong Thi Tinh, Nguyen Thu Thuy, Dinh Tran Ngoc Huy. (2021). Doing Business Research and Teaching Methodology for Undergraduate, Postgraduate and Doctoral Students-Case in Various Markets Including Vietnam, Elementary education online, 20(1).

4. Dinh Tran Ngoc Huy, Nguyen Thi Hang. (2021). Factors that affect stock price and Beta CAPM of Vietnam Banks and Enhancing Management infomation system Case of Asia Commercial Bank, Revista geintec Inovacao E Tecnologias, 11(2).

5. Das, N.M., \& Rout, B.S. (2020). Impact of COVID-19 on Market Risk: Appraisal with Value-at-risk Models, The Indian economic journal, 1.

https://doi.org/10.1177/0019466220981824

6. Dinh Tran Ngoc Huy, Pham Ngoc Van, Nguyen Thi Thu Ha. (2021). Education and computer skill enhancing for Vietnam laborers under industry 4.0 and evfta agreement, Elementary education online, 20(4).

7. Dinh Thi Hien, Dinh Tran Ngoc Huy, Nguyen Thi Hoa. (2021). Ho Chi Minh Viewpoints about Marxism Moral Human Resource for State Management Level in Vietnam, Psychology and education, 58(5).

8. Dinh Tran Ngoc Huy. (2021). Banking sustainability for economic growth and socio-economic development-case in Vietnam , Turkish Journal of computer and mathematics education, 12(2).

9. Dimitrov V, Jain PC. (2006). The Value Relevance of Changes in Financial Leverage, SSRN Working Paper

10. Emilios, A. 2015, Bank Leverage Ratios and Financial Stability: A Micro-and Macroprudential Perspective\&amp;, Working Paper No.849, Levy Economics Institute

11. Eugene FF, French KR. (2004). The Capital Asset Pricing Model: Theory and Evidence, Journal of Economic Perspectives, 18(3).

12. González, Mariano \&amp; Nave, Juan \&amp; Rubio, Gonzalo, 2018.

Macroeconomic determinants of stock market betas ,\&quot; Journal of Empirical Finance, Elsevier, vol. 45(C), pages 26-44.

13. Grabowska, A.M., \& Orlowski, L.T. (2020). Financial market risk and macroeconomic stability variables: dynamic interactions and feedback effects, Journal of Economics and Finance volume 44, pages655-669 (2020)

14. Gunaratha V. (2013). The Degree of Financial Leverage as a Determinant of Financial Risk: An Empirical Study of Colombo Stock Exchange in Sri Lanka, 2nd International Conference on Management and Economics Paper.

15. Hac, L.D., Huy, D.T.N., Thach, N.N., Chuyen, B.M., Nhung, P.T.H., Thang, T.D., Anh, T.T. (2021). Enhancing risk management culture for sustainable growth of Asia commercial bank -ACB in Vietnam under mixed effects of macro factors, Entrepreneurship and Sustainability Issues, 8(3).

16. Hang, T.T.B., Nhung, D.T.H., Hung, N.M., Huy, D.T.N., Dat, P.M. (2020). Where Beta is going-case of Viet Nam hotel, airlines and tourism company groups after the low inflation period, Entrepreneurship and Sustainability Issues, 7(3). 17. Huy, D.T.N. (2015). The Critical Analysis of Limited South Asian Corporate Governance Standards After Financial Crisis, International Journal for Quality Research, 9(4): 741-764. 
18. Huy, D.T.N. (2012). Estimating Beta of Viet Nam listed construction companies groups during the crisis , Journal of Integration and Development, 15 (1), 57-71 19. Huy, D. T.N., Loan, B. T., and Anh, P. T. (2020). Impact of selected factors on stock price: a case study of Vietcombank in Vietnam, Entrepreneurship and Sustainability Issues, vol.7, no.4, pp. 2715-

2730. https://doi.org/10.9770/jesi.2020.7.4(10)

20. Huy, D. T.N., Dat, P. M., và Anh, P. T. (2020). Building and econometric model of selected factors' impact on stock price: a case study, Journal of Security and Sustainability Issues, vol.9(M), pp. 77-93. https://doi.org/10.9770/jssi.2020.9.M(7) 21. Huy D.T.N., Nhan V.K., Bich N.T.N., Hong N.T.P., Chung N.T., Huy P.Q. (2021). Impacts of Internal and External Macroeconomic Factors on Firm Stock Pr ice in an Expansion Econometric model-A Case in Vietnam Real Estate Industry, Data Science for Financial Econometrics-Studies in Computational Intelligence, vol.898, Springer. http://doi-org-443.webvpn.fjmu.edu.cn/10.1007/978-3-030-48853$\underline{6} 14$

22. Huy, D.T.N. , An, T.T.B. , Anh, T.T.K., Nhung, P.T.H. (2021). Banking sustainability for economic growth and socio-economic development - case in Vietnam, Turkish Journal of Computer and Mathematics Education, 12(2), pp.25442553

23. Huy, D.T.N., An, T.T.B. , Anh, T.T.K., Nhung, P.T.H. (2021). Banking sustainability for economic growth and socio-economic development-case in Vietnam, Turkish Journal of Computer and Mathematics Education, 12(2), pp. 25442553

24. Hung, J.H., \& Liu, Y.C. (2005). An examination of factors influencing airline beta values, Journal of Air Transport Management 11(4):291-296.

DOI:10.1016/j.jairtraman.2005.01.004

25. Khwaja, Asim Ijaz., and Mian, Atif. (2005). Unchecked intermediaries:Price manipulation in an emerging stock market, Journal of Financial Economics 78, 243 241

26. Melicher, R.W. (1974). Financial Factors which Influence Beta Variations within an Homogeneous Industry Environment, The Journal of Financial and Quantitative Analysis, 9(2): 231-241

27. Nguyen Thi Hang, Dinh Tran Ngoc Huy. (2021). Better Risk Management of Banks and Sustainability-A Case Study in Vietnam, Revista geintec Inovacao E Tecnologias, 11(2).

28. Nguyen, T. P. L., Tran, N. M., Doan, X. H., \&amp; Nguyen, V. H. (2019). The impact of knowledge sharing on innovative work behavior of Vietnam telecommunications enterprises employees. Management Science Letters, 10(2020), 53-62.

29. Nguyen Thi Hoa, Nguyen Thi Hang, Nguyen Thanh Giang, Dinh Tran Ngoc Huy. (2021). Human resource for schools of politics and for international relation during globalization and EVFTA, Elementary education online, 20(4).

30. Pham Minh Dat, Nguyen Duy Mau, Bui Thi Thu Loan, Dinh Tran Ngoc Huy. (2020). Comparative China corporate governance standards after financial crisis, corporate scandals and manipulation, Journal of security \& sustainability issues, 9(3). 31. Pham Van Hong, Huynh Xuan Nguyen, Dinh Tran Ngoc Huy, Le Thi Viet Nga, Nguyen Thi Ngoc Lan, Nguyen Ngoc Thach, Hoang Thanh Hanh.(2021). Sustainable bank management via evaluating impacts of internal and external macro factors on lending interest rates in Vietnam, Linguistica Antverpiensia, Issue 1, pp.76-87. 32. Perkovic, A. (2011). Research of Beta As Adequate Risk Measure - Is Beta Still Alive?, Croatian Operational Research Review (CRORR), vol. 2, pp.102-111. 
33. Puspitaningtyas, Z. (2017). Estimating systematic risk for the best investment decisions on manufacturing company in Indonesia, Investment Management and Financial Innovations, vol.14, no.1, pp. 46-54. doi:10.21511/imfi.14(1).2017.05 34. Park, J.C, Ali, F.D., Mbanga, C. (2019). Investor sentiment and aggregate stock returns: the role of investor attention, Review of Quantitative Finance and Accounting, 53(2), 397 - 428.

35. Phung Tran My Hanh, Nguyen Thi Hang, Dinh Tran Ngoc Huy, Le Ngoc Nuong. (2021). Enhancing Roles of Banks and the Comparison of Market Risk and Risk Policy Implications in Group of Listed Vietnam Banks During 2 Stages: Pre and Post-Low Inflation Period, Revista geintec-gestao Inovacao e Tecnologias, Vol.11(2).

36. Satrio, A.B. (2015). CAPM and Three Factor Model: Empirical Testing From Emerging Market, Research Journal of Finance and Accounting, 6(18).

37. Uddin, M.N. , \& Ahmmed, M. (2018). Islamic Banking and Green Banking for Sustainable Development: Evidence from Bangladesh ,Jurnal Ilmu Ekonomi Syariah (Journal of Islamic Economics) Volume 10 (1) 